\title{
The University of Zurich (UZH) Hosts the Second Biannual Siegfried Symposium
}

\author{
Nathaniel S. Finney*
}

\begin{abstract}
On Thursday, September $21^{\text {st }}$, the University of Zurich hosted the second biannual Siegfried Symposium. The Symposium, which highlights new developments in process chemistry, was jointly organized by the Organic Chemistry Institute and Siegfried, Ltd. of Zofingen. A full day of lectures from a group of leading international researchers culminated in the award of the Siegfried Medal to Prof. Stephen Buchwald (MIT) for his fundamental contributions to the development of efficient transition metal-catalyzed synthetic processes.
\end{abstract}

Keywords: Organic Chemistry Institute, University of Zurich · Siegfried Medal · Siegfried Symposium

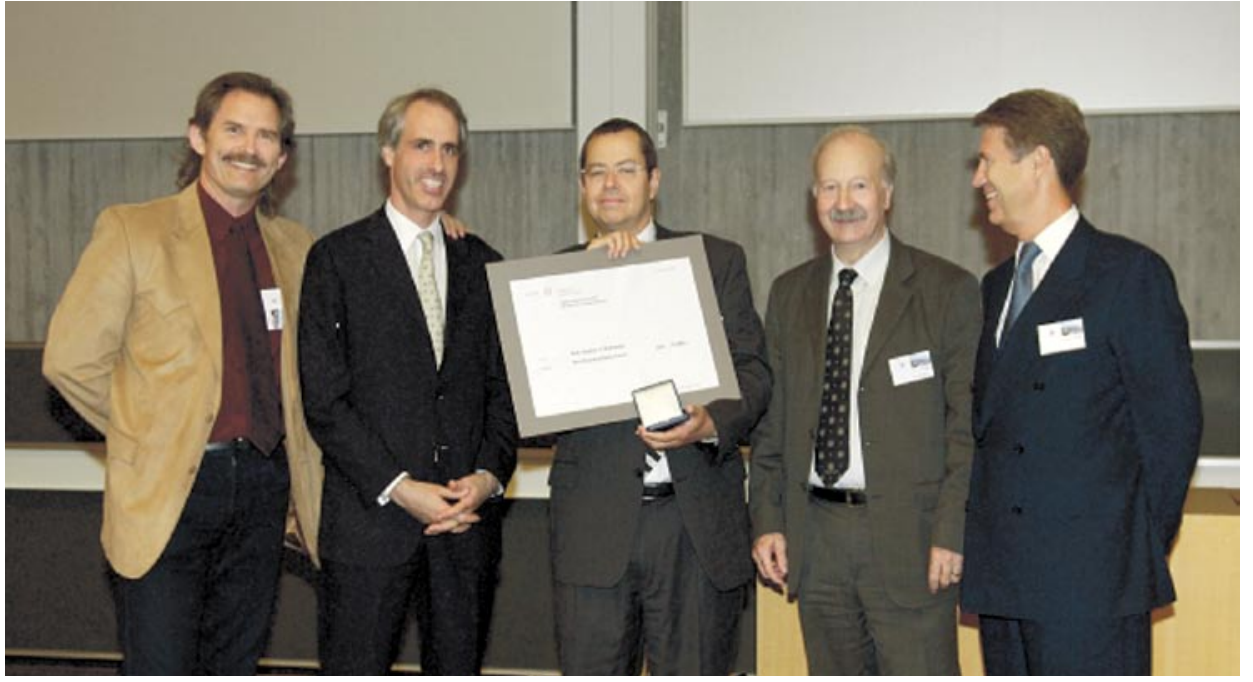

Award of the Siegfried Medal (left to right): Prof. Jay S. Siegel (Director of the OCI, UZH), Douglas Günthard (CEO, Siegfried, Inc.), Prof. Stephen Buchwald (MIT), Prof. Hans Weder (President, UZH) and Dr. Markus Altweg (Chairman of the Board, Siegfried Holding, Inc.).

${ }^{\star}$ Correspondence: PD Dr. N.S. Finney

University of Zurich

Institute of Organic Chemistry

Winterthurerstrasse 190

$\mathrm{CH}-8057$ Zurich

Tel.: + 41446354283

E-Mail: finney@oci.unizh.ch
On Thursday, Sept. 21st, the second biannual Siegfried Symposium on process chemistry was held at the University of Zurich

Professor Heini Murer, Vice President for Research at UZH welcomed the crowd with a short introduction which emphasized the role of Organic Chemistry in society and its history in Zurich. He also applauded such joint industry/university efforts as this Award Symposium in Process Chemistry, especially given the success of the Labor für Prozessforschung (LPF) run by the OCI.

The opening lecture, presented by Volker Hessel (Institut für Mikrotechnik Mainz Gmbh, DE), addressed the state of the art for the industrial application of capillary flow reactors. Such microreactors can attain flow rates of 100 1/hour, and can offer significant advantages over tra- ditional reactors in terms of size, ease of installation and use, energy consumption and waste production. However, further engineering is needed to expand the range of reaction suitable for microreactors, which have difficulty with reactions that are slow or produce solid byproducts. The following lecture, given by Olivier Ludemann-Hombourger (Novasep SAS, FR), challenged one of the central dogmata of process chemistry - that chromatography is not technically or economically viable on large scale. It was amply demonstrated that, with careful manufacturing and implementation, large-scale chromatography can be carried out by either direct scale-up of traditional HPLC columns (to sizes as large as $4 \mathrm{~m} \mathrm{~h} . \times 1.6 \mathrm{~m} \mathrm{~d}$.) or through the implementation of new technologies such as simulated moving bed (SMB) apparati.

The session continued with a presentation from Michael Lofthagen (Syntagon, $\mathrm{SE}$ ). A retrospective of the evolution of the company - which provides outsource process research \& development - highlighted the central challenges facing European process companies, namely highly variable demand and increasing competition from Asia. It was emphasized that these challenges can be met by placing a premium on speed and quality and by forming strategic relationships with emerging economies within Europe (the Balkan states and Eastern European countries). The morning session then closed with a presentation from Michael O'Brien (Wyeth Research, US), who provided an overview of Wyeth's multidisciplinary approach to process scale up and iterative optimization. The talk outlined an approach based on risk analysis and the early delineation of 'design and control 
space' - the conceptual space encompassing the critical parameters and their controllable range(s). In addition, using an analog of rapamycin as an illustrative example, the integration of process chemistry intuition with modern analytics, parallel experimentation and Design of Experiment methods was discussed, and was shown to accelerate the development of uniformly robust processes from synthesis through final API stability and formulation.

The first lecture of the afternoon was given by Roger Snowden (Firmenich, $\mathrm{CH}$ ). Following an initial demonstration of the dependence of jasmonate ester odor on diastereomeric and enantiomeric identity, recent synthetic advances in jasmonate synthesis were described; these included a new system for enantio- and diastereoselective Ru-catalyzed hydrogenation and a remarkable diastereoselective Claisen rearrangement. The talk finished with a discussion of recent synthetic studies on Ambrox (originally prepared from ambergris) based on an elegant acid-catalyzed polyene cyclization. This was followed by a presentation from David Askin (Merck \& Co. Inc., US), which began with an overview of the evolution of the manufacturing process for the important HIV protease inhibitor indinavir. Multiple synthetic approaches were described, with a detailed description of strategy, yields and impurity profiles. Notably, the final process uses two components synthesized by the industrial-scale application of catalytic enantioselective processes. This was followed by recent developments in the synthetic process for a new HIV integrase inhibitor, which provided an impressive example of 'through process' chemistry (or 'telescoping'), by which a process is designed to allow several sequential steps to be carried out without isolation or separate purification of the intermediates. The penultimate talk of the afternoon featured Jean-Michel Adam (Hoffmann-La Roche, CH). It initially focused on the preparation of a new Factor VIIa inhibitor, which has promise for the treatment of deep vein thrombosis and the prevention of stroke. A series of different strategies for the efficient synthesis of a key $\alpha$-aryl amino acid were described, which embodied a strategy of iterative process development during the transitions from discovery to pre-GMP to early GMP to final API production. The second portion of the talk discussed the strategic use of laboratory automation in process development. Illustrating with a series of examples, a mixed approach to automation implementation was described, emphasizing a balance between lower-throughput methods that could be easily adopted by bench chemists and higher-throughput methods that rely on centralized equipment and require (more) specialized training.

The Symposium culminated with the awarding of the Siegfried Medal by UZH President Hans Weder and Siegfried Holdings Chairman Markus Altweg to Prof. Stephen Buchwald (Massachusetts Institute of Technology, US) for his numerous contributions to the development of robust and practical transition metal-catalyzed reactions. Prof. Buchwald's lecture covered three main areas: optimized Pd catalyst systems for $\mathrm{C}-\mathrm{N}$ bond formation; related catalyst systems for $\mathrm{C}-\mathrm{C}$ bond formation; and recent developments in the $\mathrm{Cu}$-catalyzed formation of $\mathrm{C}-\mathrm{N}$ bonds. Each section emphasized the interplay between ligand optimization and mechanistic understanding in these important reactions, and provided a clear summary of the current state of the art. The Award lecture was followed by a lively and well-attended Apéro.

UZH and the OCI gratefully acknowledge the financial and logistical support for the Symposium provided by Siegfried, Inc. Digital video of the Symposium lectures may be found on the OCI website (http:// www.oci.unizh.ch/diversa/SiegfriedSymposium2006/index.html)

Received: October 20, 2006 